\title{
Aoristo)))))
}

International Journal of Phenomenology, Hermeneutics and Metaphysics

\section{Antropologia e metafisica in Edmund Husserle Edith Stein}

\author{
Anthropology and metaphysics in Edmund Husserl and \\ Edith Stein
}

\author{
Prof.essa. Dr.essa Angela Ales Bello \\ Pontificia Università Lateranense di Roma'
}

\section{RIASSUNTO}

Il mio scopo in questo articolo è quello di affrontare le analisi di E. Husserl ed E. Stein sulle questioni antropologiche e metafisiche. Per svolgere questo compito è necessario spiegare il significato del metodo fenomenologico, prima in Husserl e poi in E. Stein, sottolineando la novità del loro approccio alla conoscenza dell'essere umano, del mondo e di Dio. Il saggio è diviso in quattro parti; due sono dedicati all'indagine di Husserl e Stein sull'essere umano e due al loro sviluppo di temi metafisici. Per quanto riguarda il primo argomento, si scopre una significativa connessione tra i due pensatori; per quanto riguarda il secondo, si nota che è possibile ritrovare un approccio metafisico alla realtà anche in Husserl e uno sviluppo originale del tema nell'analisi di Stein legata alla filosofia medievale e alla teologia.

\section{PAROLE CHIAVE}

Metodo fenomenologico; Gnoseologia; Antropologia; Indagine metafisica dell'essere umano; Approccio metafisico a Dio

\section{ABSTRACT}

My aim in this paper is to deal with E. Husserl's and E. Stein's analyses on the anthropological and metaphysical questions. To perform this task it is necessary to explain the meaning of the phenomenological method, first of all in Husserl and then in E. Stein, underscoring the novelty of their approach to the knowledge of human being, world and God. The essay is divided in four parts; two are dedicated to Husserl's and Stein's investigation on the human being and two to their

\footnotetext{
${ }^{1}$ E-mail: alesbello@tiscali.it, Orcid: https://orcid.org/0000-0001-8929-1307
} 


\section{Aoristo)))))}

International Journal of Phenomenology, Hermeneutics and Metaphysics

development of metaphysical themes. Regarding the first topic we discover a significant connection between the two thinkers, regarding the second one we notice that it is possible to find a metaphysical approach to reality even in Husserl and an original development of the theme in Stein's analysis linked up with the medieval philosophy and theology.

\section{KEYWORDS}

Phenomenological method; Gnoseology; Anthropology; Metaphysical investigation of human being; Metaphysical approach to God

\section{INTRODUZIONE}

I due filosofi che intendo prendere in considerazione sono legati, com'è noto, da un rapporto di discepolato dell'una nei confronti dell'altro e rappresentano nella filosofia contemporanea due momenti distinti e connessi che li rendono originali e importanti per il loro contributo teoretico.

Husserl è un pensatore, il quale fornisce alla storia della filosofia occidentale un nuovo punto di vista, aggiungendo a ciò che era stato detto la possibilità di porsi in una nuova prospettiva, non per mettere in crisi tutto ciò che di valido era stato proposto, piuttosto per chiarire, appunto, alcuni aspetti, che a suo avviso, erano già presenti, ma non erano stati adeguatamente evidenziati. Edith Stein comprende tale novità e completa l'inserimento della fenomenologia husserliana nella storia del pensiero occidentale, ripercorrendolo nelle sue linee essenziali e stabilendo contatti con il pensiero antico e, soprattutto, con quello medievale.

In quest'occasione mi fermerò a evidenziare la novità del metodo fenomenologico e i suoi risultati nel campo gnoseologico, per procedere alle sue applicazioni nell'ambito dell'antropologia filosofica e dei temi metafisici della tradizione occidentale.

\section{L'ANTROPOLOGIA FILOSOFICO-FENOMENOLOGICA DI HUSSERL}

Il titolo assertivo di questo paragrafo è già una sfida, perché si può sollevare subito un'obiezione: esiste un'antropologia filosofica in Husserl?

Osservo preliminarmente che "antropologia" si dice in molti modi. Le diverse definizioni di antropologia sono indicate con grande precisione da Edith Stein: ella distingue l'antropologia come "scienza naturale" che si riferisce all'essere umano inteso come un essere della natura, l'antropologia come "scienza dello spirito", che mette in evidenza l'attività spirituale umana, secondo l'impostazione di Dilthey, Rickert, Windelband, e, infine, l'antropologia filosofica (STEIN, 2013). Anche Husserl aveva rilevato tali differenze in due testi contenuti nelle raccolte dei suoi scritti, rispettivamente in Aufsätze und Vorträge (1911-1921) e in Aufsätze und Aufträge (19221937), testi emblematici in riferimento all'argomento che stiamo trattando, perché riguardano i diversi ambiti d'indagine, ai quali il termine antropologia si riferisce. 


\section{Aoristo)))))}

International Journal of Phenomenology, Hermeneutics and Metaphysics

Il primo è Phänomenologie und Psychologie (HUSSERL, 1987), la cui stesura risale al 1917 e conclude un periodo cruciale per la delineazione della fenomenologia di Husserl.

Il testo si apre con una perentoria affermazione secondo la quale il "fenomeno" essere umano, che gli interessa indagare, non deve essere confuso con quello di cui parlano le scienze naturali.

La fenomenologia è, piuttosto, una scienza "parallela" che si muove sul piano eidetico e, rispetto ai campi del sapere presi nella loro globalità, cerca di comprendere quale sia l'apriori che li caratterizza, in altri termini, quale sia l'"idea", cioè il punto essenziale della soggettività animale, della persona e delle associazioni umane, che sono alla base delle discipline empiriche come la psicologia, l'antropologia e le varie scienze dello spirito; altrettanto vale, come Husserl aveva indicato nel primo volume delle Idee per una fenomenologia pure e una filosofia fenomenologica (HUSSERL, 2002), per le scienze della natura. Si tratta, pertanto, di analizzare quelle che egli definisce "ontologie regionali", le quali ruotano intorno a un fenomeno di cui si cercano le specificazioni, presupponendo che ci sia una struttura essenziale di quel territorio. In quest'ottica, anche l'antropologia è un'ontologia regionale. Si può osservare che la delineazione delle ontologie regionali corrisponde a quello che definisco il primo passo del metodo fenomenologico, cioè la riduzione all'essenza, seguita dal secondo passo, consistente della riduzione fenomenologico-trascendentale, alla quale si farà riferimento in seguito. Per ora è opportuno riproporre brevemente il ragionamento di Husserl contenuto nel testo citato.

Egli prosegue affermando che l'indagine fenomenologica rappresenta un terzo momento rispetto alle scienze della natura e di quelle dello spirito, proponendosi come una nuova scienza (eine völlige neue Wissenschaft) di tipo filosofico e, per raggiungere il suo peculiare statuto epistemologico, essa deve mettere fuori circuito tutte le scienze già costituite, come Husserl ripete più volte. In particolare nel $\S 56$ delle Idee I Husserl scrive: “Dal punto di vista della scienza fenomenologica da fondare si tratta di stabilire da quali scienze la fenomenologia possa liberamente attingere senza perdere la purezza del suo 'senso' [...]. Ed è proprio dell'essenza della fenomenologia quale scienza delle 'origini' di porsi e cautamente ponderare simili questioni metodologiche, ignorate dalle scienze ingenue ('dogmatiche')" (HUSSERL, 2002, p. 142). Il risultato è che sono messe fuori circuito tutte le scienze naturali e le scienze dello spirito, quindi, anche quelle che si riferiscono all'umano; allora, come proseguire nella ricerca? Ciò che rimane fuori dalla messa tra parentesi è la "struttura conoscitiva dell'io" presa nella sua purezza. Ma prima di esplicitare tutto ciò è opportuno riferirsi al secondo testo in cui Husserl affronta la questione dell'antropologia.

Egli torna a riflettere su quest'argomento dopo più di dieci anni, quando ormai il suo metodo è del tutto configurato, in uno scritto degli anni Trenta, Phänomenologie und Anthropologie. Si tratta di una conferenza tenuta nelle Società kantiane di 


\section{Aoristo)))))}

\section{International Journal of Phenomenology, Hermeneutics and Metaphysics}

Francoforte, Berlino e Halle nel 1931, conferenza che egli dedica, appunto, al rapporto fra fenomenologia e antropologia 2 .

All' inizio del suo saggio Husserl riconosce che nella generazione più giovane si manifesta un interesse per l'antropologia filosofica, ma quest'ultima s'identifica con la "filosofia della vita" di Dilthey contro la quale egli ha polemizzato fin dal famoso articolo La filosofia come scienza rigorosa (1910-11), accusandola di relativismo e nota che l'influenza diltheyana è presente anche nei giovani fenomenologi. Ci si può domandare se egli si riferisca all'esplicito richiamo a Dilthey fatto da Heidegger in Essere e Tempo (HEIDEGGER, 1953) ${ }^{3}$. Ciò sembrerebbe confermato dall'affermazione di Husserl secondo la quale, mentre la fenomenologia era nata in opposizione all'antropologismo e allo psicologismo, sembra che la filosofia fenomenologica debba essere interamente ricostruita movendo dall' "esserci umano" (menschlichen Dasein) 4 Com'era, in effetti, nel progetto heideggeriano, e questo, secondo Husserl è una forma di antropologismo, perché non passa attraverso la riduzione fenomenologica. Comprendiamo anche attraverso tale affermazione la ragione del contrasto fra i due filosofi e della rottura dei loro rapporti intellettuali e umani.

La cosa interessante è che l'accusa di antropologismo era già stata mossa da Heidegger a Husserl; infatti, nel $\$ 10$ della Prima Parte di Essere e Tempo Heidegger accomuna Husserl e Scheler, sostenendo che nella loro indagine fenomenologica, benché più radicale di altre forme di personalismo, “[...] non pongono l'essere della persona in quanto tale" (HEIDEGGER, 1953, p. 60).

Che cosa intendono Husserl e Heidegger per " antropologismo"? Il primo ritiene che, se si muove dall'analisi del menschlichen Dasein e non si compie un'epoché radicale, si rimane su un terreno naturalistico, il secondo, al contrario, sostiene che i risultati ai quali si giunge attraverso le riduzioni fenomenologiche husserliane sono legati a una visione tradizionale, alla quale contrappone una nuova analitica esistenziale.

Nella conferenza ora citata, in realtà, Husserl non solo non condivide il modo, a suo avviso, improprio di usare la fenomenologia da parte di alcuni, ma assimila l'antropologia filosofica, contro la quale polemizza, a quella di origine diltheyana, definendola antropologismo e psicologismo, dove gli 'ismi' stanno a indicare un'assolutizzazione arbitraria e, quindi, una deviazione da una retta indagine; la sua critica è rivolta, quindi, da un lato, all'analitica esistenziale, dall'altro, anche, come lo era già nella Filosofia come scienza rigorosa, alla tentazione di consegnare l'indagine sull'essere umano alla psicologia (HUSSERL, 2016).

A tutto questo Husserl contrappone una ricerca filosofica che ha come suo nucleo e suo centro la dimensione trascendentale. E qui egli descrive e utilizza quello che definisco il secondo passo del metodo, appunto, la riduzione trascendentale.

Dato l'ambiente nel quale la conferenza viene pronunciata - come si è visto si tratta di Società kantiane - si potrebbe supporre che l'uso del termine

\footnotetext{
${ }^{2}$ E. Husserl, Phänomenologie und Anthropologie, in Aufträge und Vorsätze (1922-1937), Husserliana, Bd. XXVII.

${ }^{3}$ M. Heidegger, Sein und Zeit, Zweiter Abschnitt, V Kapitel, § 77.

4 “ Die Phänomenologische Philosophie soll völlig neu von menschlichen Dasein her aufgebaut werden"(E. Husserl, Phänomenologie und Anthropologie, cit. p.164.
} 


\section{Aoristo)))))}

\section{International Journal of Phenomenology, Hermeneutics and Metaphysics}

"trascendentale" conduca a un'apertura nei confronti della posizione di Kant; ma egli stabilisce la sua distanza da quest'ultimo, facendo riferimento, piuttosto, a Cartesio, rispetto al quale sottolinea la sua vicinanza e contemporaneamente la sua distanza. Com'è noto, il rapporto con Cartesio è costante fin dal primo volume delle Idee per una fenomenologia pura, culmina nelle Meditazioni cartesiane (HUSSERL, 2017) e sarà riaffrontato nelle conferenze di Vienna e di Praga del 1934, che costituiranno il nucleo della Crisi delle scienze europee e la fenomenologia trascendentale (HUSSERL, 1987) - per citare solo le opere edite più note.

Cartesio è considerato come l'iniziatore della svolta filosofica dell'Età Moderna, quella che conduce a stabilire il nuovo punto di partenza della filosofia nella soggettività, o meglio nella sfera egologica. Tuttavia, numerose sono le distinzioni proposte da Husserl rispetto all'analisi cartesiana dell'ego. Se egli condivide il punto partenza, si può costatare che né il procedimento seguito per raggiungerlo né i risultati ottenuti dal filosofo francese siano da Husserl accettati. In primo luogo, egli pone una profonda distinzione fra la sua posizione e il dubbio cartesiano che, mettendo in forse l'esistenza del mondo, supera ciò che è nelle possibilità dell'essere umano - il mondo non può essere negato, è tanto presente nella sua esistenza che non può essere messa in dubbio, eventualmente esso può solo essere messo fra parentesi e Husserl, da matematico oltre che da filosofo, ricorda al matematico e filosofo Cartesio che tutto ciò che è messo in parentesi "vive" nella parentesi (HUSSERL, 1987) 5 .

L'epoché, pertanto, è solo un cambiamento di atteggiamento che dall'ingenuità dell'accettazione naturale di tutto ciò che ci viene incontro, comprese le scienze già costituite, conduce al rivolgimento di prospettiva che caratterizza l'approccio teoretico della filosofia occidentale. E ciò che rimane non è l'essere umano nella sua solitudine, ma la solitudine dell'ego trascendentale subito riempita, come si ricorderà nella Quinta delle Meditazioni cartesiane, dalla presenza dell'alter-ego.

Allora che cosa è l'ego trascendentale? Esso, se bene inteso, è tale, secondo Husserl, da superare l'obiezione radicale consistente nel dire che se l'io, cioè questo essere umano (Mensch) esercita la metodica della presa di posizione trascendentale e, in tal modo, ritorna al suo puro ego, allora l'io non è altro che un livello astratto dell'essere umano concreto, il suo puro spirito, dal momento che è astratto rispetto al corpo e ciò era sostenuto da Cartesio. Chi sostiene ciò, però, secondo Husserl, ricade in un atteggiamento ingenuo e naturale e dimostra che il suo pensiero si muove sul terreno del mondo predato invece che nell'ambito dell'epoché: considerarsi come essere umano, in ciò consiste la presupposizione della validità del mondo. Attraverso l'epoché, la messa fra parentesi di tutte le conoscenze già date e per ricominciare la ricerca, diventa chiaro che è l'ego colui nella cui vita l'appercezione essere umano è mantenuta all'interno dell'universale appercezione del senso d'essere del mondo ${ }^{6}$.

\footnotetext{
${ }^{5}$ Ivi, §31.

${ }^{6}$ E. Husserl, Phänomenologie und Anthropologie, cit., p.164.
} 


\section{Aoristo)))))}

International Journal of Phenomenology, Hermeneutics and Metaphysics

La questione consiste, allora, nel chiedersi se la riduzione all'ego elimini il Mensch come Mensch in der Welt, l'essere umano come essere nel mondo, secondo la terminologia heideggeriana. Husserl si affretta a rilevare nella conferenza in esame che il mondo rimane un tema fondamentale e non è eliminato ma sottratto alla "ingenuità" della conoscenza quotidiana. In questo testo egli è maggiormente impegnato a garantire la possibilità di giustificare la correlazione fra il mondo e il soggetto, piuttosto che ad analizzare la struttura dell'essere umano, che emerge, in ogni caso, proprio dall'approfondimento della dimensione trascendentale. Tale struttura era già stata indagata nel vol. II delle Idee per una fenomenologia pura trascritto dagli appunti stenografici husserliani da Edith Stein; fra le opere edite questo è, infatti, il testo più completo per la delineazione di un'antropologia filosofica.

Si tratta, allora, di un nuovo modo di condurre l'analisi trascendentale che consente di individuare il fluire di vivenze ${ }^{7}$ (Erlebnisse), di cui si ha "coscienza" da intendersi come strumenti di comprensione di tutte le dimensioni del soggetto - è questa la novità dell'analisi fenomenologica rispetto alle acquisizioni gnoseologiche dalla filosofia occidentale; infatti, descritti essenzialmente, tali vivenze rimandano alle strutture "reali" dell'essere umano. Il primo e il secondo volume delle Idee per una fenomenologia pura sono, in tal modo, connessi e devono essere letti nel loro reciproco rimando. Se il primo è rivolto a dare le connotazioni del metodo e l'ambito dell'analisi, quello, appunto, della dimensione trascendentale come luogo di svelamento del senso della realtà quoad nos, il secondo è rivolto a mettere in luce la costituzione della natura materiale alla quale appartiene il corpo, cioè la natura animale, caratterizzata dalla realtà psichica, e il mondo dello spirito, al quale appartiene l'io personale.

$\mathrm{E}^{\prime}$ interessante notare come, dopo aver messo tra parentesi tutte le dottrine tradizionali riguardanti l'essere umano si affronti, in modo originale, perché non deduttivo ma ostensivo, l'analisi del fenomeno essere umano, recuperando e avvalorando la sua tripartizione di lontana origine paolina in corpo, psiche e spirito.

Si può sottolineare come la nozione di Leib, corpo vivente, sia squisitamente fenomenologica, infatti, la struttura dell'essere umano si può mostrare iniziando dall'esame del corpo vivente, il quale non è di per sé un punto di partenza, ma è rintracciato nelle sue caratteristiche movendo dalla presenza della "percezione" come un vissuto di cui si ha coscienza. Se l'apprensione percettiva presuppone $\mathrm{i}$ contenuti della sensazione, i quali svolgono un ruolo necessario per la costituzione degli schemi e così per la costituzione delle apparizioni delle cose stesse reali, ciò "comporta che in tutte le percezioni, in tutte le dimostrazioni fornite dalla percezione (esperienza) è presente il corpo vivo in quanto organo di senso liberamente mobile, in quanto totalità liberamente mobile degli organi di senso, e perciò che, in virtù di

\footnotetext{
${ }^{7}$ Utilizzo questo termine in assonanza con vivencia presente nella lingua spagnola e portoghese, perché mi sembra che esprima più validamente il fatto che stiamo vivendo una certa esperienza, mentre la traduzione ufficiale italiana è "vissuto", tratto dalla lunga espressione: "ciò che è da noi vissuto", ma, se isolato, questo vocabolo, vissuto, indica più qualcosa di passato, che di "passivo".
} 


\section{Aoristo)))))}

International Journal of Phenomenology, Hermeneutics and Metaphysics

questo fondamento originario, qualsiasi cosa, qualsiasi realtà del mondo circostante dell'io ha una propria relazione con il corpo vivo " (HUSSERL, 2000, p. 60).

Una volta individuato il corpo vivente nel secondo volume delle Idee, da cui ho tratto la citazione precedente, non ci si aspetterebbe una ripresa del tema della coscienza attraverso l'io puro, da intendersi come il punto di riferimento essenziale della corrente dei vivenze, che Husserl aveva già descritto nel primo volume; ma tale ripresa serve a ricordare che la descrizione di esso nei suoi tratti essenziali è possibile grazie a tale capacità dell'essere umano scoperta attraverso la riflessione; pertanto "[...] questo io non è né misterioso, o addirittura di mistico. Io prendo me stesso in quanto io puro, mi prendo cioè puramente come ciò che nella percezione è diretto verso il percepito, nel conoscere verso il conosciuto, nel fantasticare sul fantasticato, nel pensiero logico verso il pensato [...] In termini più precisi: l'io puro è in riferimento con gli oggetti in modi molto diversi, secondo il genere dell'atto che compie" (HUSSERL, 2000, p. 102-103).

Tali atti, colti nella loro universalità, possono essere quelli del desiderio, dell'amore, dell'odio, dell'attrazione e della ripugnanza, della decisione nell'azione, inoltre l'atto del fiat, della volontà, e ancora gli atti teoretici del delineare un contesto tematico, dello stabilire relazioni, di porre un soggetto e un predicato, di trarre conseguenze. Si è rintracciata, in tal modo, una struttura trascendentale sui generis che consente di passare all'indagine dell'essere umano in quanto natura - il corpo vivente come latore di sensazioni localizzate - oppure come attraversato dalle sensazioni di piacere, di dolore, di benessere o di disagio che costituiscono la base materiale, la base che egli definisce hyletica, intendendo con essa la base per la costituzione dei valori, quindi con questo strato originario si connettono le funzioni intenzionali e i materiali assumono una funzione spirituale.

Attraverso lo strato di qualità 'reali' (base hyletica) - in quanto si costituiscono in virtù di una relazione con circostanze reali nell'ambito del reale, il corpo vivente s'intreccia con la psiche, pertanto, si può affermare che:

La psiche e l'io psichico 'hanno' un corpo vivo; esiste una cosa materiale di una natura tale che essa non è mera cosa materiale, bensì appunto, corpo vivo, e quindi una cosa materiale che in quanto campo di localizzazioni di sensazione e di moti del sentimento, in quanto complesso di organi di senso, in quanto elemento fenomenico e controparte di qualsiasi percezione di cose, [...] è una base fondamentale della datità reale della psiche e dell'io (HUSSERL, 2000, p. 159).

Si è giunti, allora, a un'altra dimensione qualitativamente diversa dalla cosa materiale, cioè la psiche, ma il corpo vivente è, appunto, l'intreccio di questi due momenti. Alcuni manoscritti husserliani analizzano, scavando ulteriormente nella dimensione psichica, l'ambito dell'istinto, che indica una continuità con il mondo 


\section{Aoristo)))))}

\section{International Journal of Phenomenology, Hermeneutics and Metaphysics}

animale; tuttavia, la distinzione con tale mondo si rintraccia nella funzione intenzionale e spirituale (ALES BELLO, 2000).

Si è visto sopra che all'io puro fanno capo anche vivenze che sono diversi dalle tensioni, dagli impulsi, dalle reazioni, questi sono gli atti volontari, valutativi e teoretici che caratterizzano la persona umana; si entra, in tal modo, nella vita dello spirito che non è in alcun modo "determinata", ma "motivata", essa è la sede degli atti liberi e delle prese di posizione razionali. Passività e attività s'intrecciano, ma l'attività distingue l'essere umano "desto", desto eticamente e teoreticamente.

Si procede, pertanto, alla definizione della persona, che è tale, appunto, se ha rappresentazioni, sente, valuta, persegue qualche cosa, agisce, e in ciascuno di questi atti personali è in relazione con qualche fine, con gli oggetti del suo mondo circostante.

Assumere un atteggiamento personalistico nei confronti del mondo circostante significa assumere un atteggiamento valutativo ed etico. Questo, lungi dall'essere artificiale, è il vero e proprio atteggiamento "naturale". Si tratta di uno dei pochi luoghi in cui il termine "naturale" è utilizzato da Husserl, per solito egli lo esclude perché lo assimila all' uso che ne fa il Positivismo nell' ambito del naturalismo.

Certamente la posizione husserliana non è una posizione sostanzialista, tutto ciò è lontano dal suo orizzonte mentale per una serie di ragioni: la sua formazione scientifica, il suo accostarsi alla filosofia in modo personale, senza legami con una particolare corrente di pensiero, la sua appartenenza al mondo culturale protestante in polemica con la filosofia medievale e per il suo rifiuto della metafisica razionalistica dell'Età Moderna. Tuttavia egli, com'è noto, è introdotto alla filosofia attraverso Brentano, ex-sacerdote cattolico, filosofo erede della tradizione antica e medievale nell'area austriaca rimasta fedele alla Chiesa di Roma, e sostenitore di una filosofia rigorosa benché aperta alle nuove istanze della psicologia ${ }^{8}$.

Edith Stein afferma che tutto ciò non è secondario per comprendere la descrizione "essenziale" dell'essere umano proposta da Husserl, essenzialità che non si muove immediatamente in uno sfondo metafisico, ma che consente di delineare l'essere umano nelle sue caratteristiche proprie, che consentono di affermare la sua eccedenza rispetto a ciò che è "naturale". Il risultato dopo l'epoché di tutte le interpretazione già date, è, in verità, il recupero della tradizione occidentale grecocristiana: la fenomenologia, in quanto filosofia fenomenologica, approda a una descrizione che avvalora la tradizione, ma lo fa seguendo un nuovo percorso.

Nell'antropologia filosofica husserliana - possiamo, in effetti, usare ormai questa espressione, - , un'antropologia delineata su un terreno fenomenologico e quindi, come ho già indicato, ostensivo e non speculativo, grande spazio è dedicato alla dimensione etico-religiosa. Prevalente è l'aspetto gnoseologico nella ricerca husserliana e certamente esso è importante come via per la soluzione di tutti i problemi, ma egli non ha accantonato soprattutto nella sua ricerca privata i "problemi ultimi e sommi" che definisce "metafisici" come afferma in una delle sue opere più importanti le Meditazioni cartesiane. La questione etico - religiosa si lega alla

\footnotetext{
${ }^{8}$ Per quest'argomento rimando al mio già citato "Il senso dell'umano ecc." .
} 


\section{Aoristo)))))}

International Journal of Phenomenology, Hermeneutics and Metaphysics

questione di Dio affrontata in numerosi punti della sua opera, come ho cercato di mostrare altrove. Mi sembra importante citare questi aspetti della sua antropologia dalla quale emerge un essere umano esaminato in tutte le sue potenzialità, non ridotto solo ad alcune delle sue dimensioni, aperto agli altri - si pensi alle analisi husserliane sull'intersoggettività, ma potremmo aggiungere, sulla base di quanto è stato detto sopra, all'inter-personalità - e aperto all' Altro come giustificazione ultima della sua esistenza. Riprenderò quest' argomento poi.

La posizione husserliana sull' antropologia è avvalorata e confermata, come si è indicato sopra, proprio dalle stesse obiezioni che Heidegger muove a Husserl e anche a Scheler, accomunato quest'ultimo con il primo come bersaglio critico. Sempre nel § 10 di Essere e Tempo si legge che l'interpretazione dell'essere umano come unità corporea - animata - spirituale, quella husserliana e scheleriana appunto, è assolutamente insufficiente, perché non è possibile concepire questo essere congiungendo modi di essere come il corpo, l'anima e lo spirito, che, oltre a tutto, sono assunti come totalmente indeterminati nel loro essere. Inoltre, un tentativo d'indagine ontologica del genere sarebbe costretto a supporre un'idea dell'essere del tutto e aggiunge in modo significativo che tutto ciò è legato all'orientamento dell'antropologia greco - cristiana, il quale ha coniugato la definizione dell'essere umano come animal rationale con l'essere e l'essenza di ordine teologico.

In questa sede non interessa sviluppare che cosa Heidegger contrapponga, ma solo porre l'accento che quella che per lui era un'accusa si rivela come la connotazione fondamentale dell' antropologia proposta da Husserl.

Vorrei concludere la breve sintesi di quella che definisco l'antropologia filosofico - fenomenologica di Husserl, sottolineando che è possibile rispondere in modo affermativo alla domanda posta all'inizio di questo percorso. Anche se Husserl non ha accettato la definizione di "antropologia filosofica" per indicare il cammino compiuto e i risultati raggiunti, ha mostrato, attraverso un'analisi che muove dal soggetto umano e torna sul soggetto umano stesso - il che presuppone, come egli stesso indica, la paradossalità di questa entità, l'unica che sia capace di essere contemporaneamente soggetto e oggetto dell'indagine - in modo non assertivo, ma il più ostensivo possibile la complessità della sua struttura.

\section{IL METODO FENOMENOLOGICO E L'ANALISI DELLA PERSONA UMANA IN EDITH STEIN}

Al contrario di Husserl Edith Stein accetta da Scheler l'espressione "antropologia filosofica", seguendo, però, prevalentemente le orme del maestro Husserl nella descrizione dell'essere umano, soprattutto nella prima fase delle sue indagini che possiamo definire "fenomenologica".

Vorrei mostrare la centralità dell'analisi dell'essere umano per la nostra pensatrice; infatti, questo è l'oggetto fondamentale nella sua ricerca, l'argomento intorno al quale si affatica, la questione incessantemente presa in esame da lei fin dalla sua dissertazione di laurea Il problema dell'empatia (STEIN, 1998). La sua 


\section{Aoristo)))))}

\section{International Journal of Phenomenology, Hermeneutics and Metaphysics}

curiosità intellettuale la spinge verso la comprensione dell'altro in rapporto a se stesso e, quindi, la sollecita a rintracciare le strutture profonde che sono alla base della comune costituzione umana. Ma vorrei anche soffermarmi, ancora una volta per approfondire maggiormente l'andamento dell'analisi, sullo spirito della ricerca fenomenologica che ella condivide e che le consente di ottenere i risultati di cui parlerò in seguito. Seguiamo, in tal modo, il tipo d'indagine propria dei fenomenologi che procede per cerchi concentrici, specificando a ogni cerchio successivo ciò che era contenuto in quello precedente.

Questa ricerca, inaugurata come si è detto da Husserl, è caratterizzata da una grande attenzione rivolta alla realtà che ci circonda e a noi stessi. I fenomeni che noi incontriamo, da intendersi come "manifestazioni" che ci sono offerte, si presentano con una loro essenza che è possibile rintracciare.

Si tratta di una visione che potremmo definire ottimistica, ma che può e deve essere confermata e verificata attraverso i risultati della ricerca e non presupposta $a$ priori, secondo la quale l'essere umano riesce in una certa misura a cogliere il "senso delle cose".

Il termine "cosa" ha una pluralità di significati, non si riferisce solo alla cosa in senso fisico - nella lingua tedesca Ding -, ma anche alla Sache, che è la questione, cioè l'argomento, il fatto che deve essere compreso e fra i fatti, quindi i fenomeni che incontra, Husserl privilegia il soggetto umano. Si tratta di un lavoro di scavo non solo nella nostra singolarità, ma, movendo da essa, nella comune umanità che troviamo in noi e negli altri.

E' necessario rilevare, nell'ambito dell'analisi fenomenologica, l'importanza del momento della "scoperta", della messa in evidenza, del fatto che nulla è già dato per scontato o presupposto, che è indispensabile mettere fra parentesi ogni definizione dell'essere umano già configurata, sia dal punto di vista della tradizione filosofica, sia da quello delle scienze della natura e delle scienze umane - , come si è visto, Husserl preferiva l'espressione diffusa nel suo ambiente filosofico di scienze dello spirito. La ragionevolezza di quest'operazione di epochè, di sospensione del giudizio, di riduzione, sta nel fatto che ogni soluzione, anche se lo pretende, non può proporsi come assoluta, come definitiva e indubitabile, suscitando sempre domande e problemi. Il ricominciare da capo ha, quindi, il senso di vagliare se ciò che si dice, è detto perché così si mostra la cosa presa in esame, nella sua essenza, cioè nelle sue caratteristiche strutturali, poste in risalto attraverso uno sguardo purificato in modo tale da cogliere intuitivamente ciò che è dato non fermandosi alla superficie, ma procedendo a un lavoro di scavo. In questo consiste la garanzia del risultato.

Ispirandosi alle indagini che il suo maestro Husserl stata conducendo, in particolare quelle confluite nel I volume delle Idee per una fenomenologia pura e una filosofia fenomenologica (1913) Edith Stein sceglie come tema della sua Dissertazione di laurea, discussa nel 1916 presso l'Università di Gottinga, quello relativo alla conoscenza dell'altro approfondendo il significato di un particolare atto che compiamo costantemente, quello denominato Einfühlung, che possiamo tradurre con il termine entropatia o empatia. 


\section{Aoristo)))))}

\section{International Journal of Phenomenology, Hermeneutics and Metaphysics}

Come si è già fatto notare, nel suo lavoro di scavo all'interno della soggettività Husserl esplora proprio quel terreno che già Kant aveva individuato e definito con il termine trascendentale, ma lo scavo conduce Husserl a risultati diversi da quelli kantiani individuando, come si è già detto, gli atti che noi viviamo, quindi che sono da noi "vivenze" - così si traduce in italiano la parola tedesca Erlebnisse - che caratterizzano la nostra "interiorità"; quest' ultima espressione non è husserliana, ma la utilizzo solo per far comprendere quale sia la sfera che è presa in esame. I vivenze, o atti, sono elementi strutturali, che noi tutti possediamo, mentre i loro contenuti possono essere estremamente soggettivi, relativi, mutevoli.

All'inizio della sua Dissertazione Edith Stein riprende le linee fondamentali del metodo husserliano distinguendo le caratteristiche essenziali dei vari vivenze. Per comprendere ciò approfondiamo il percorso tracciato da Husserl. Egli inizia da un atto che considera molto importante perché ci mette in correlazione con il mondo esterno, che è l'atto della percezione. Esaminandolo in modo essenziale in se stesso e cercando di capire in che cosa consista in quanto atto, si comprende che esso ci pone in contatto diretto, immediato con la realtà, è un atto che tutti in condizioni normali possiamo compiere, ma il contenuto della percezione è mutevole, perché dipende dalle circostanze e dagli oggetti percepiti.

Nel compiere tale analisi, tuttavia, lo abbiamo isolato poiché lo viviamo insieme con una serie di atti di cui abbiamo consapevolezza; è la coscienza di tali atti che ci accompagna - la Stein la descrive come una luce che illumina i vivenze - ma ancora non tematizziamo tali atti, per fare questo abbiamo bisogno di un vissuto ulteriore e particolare che è quello della riflessione. Esso ci consente di porci nei confronti degli atti che viviamo con un atteggiamento di secondo grado per coglierne il significato.

Se analizziamo ciò che accade in noi stessi ci rendiamo conto che non solo è presente l'atto del percepire, ma anche quello del ricordare, dell'immaginare, del fantasticare, del pensare e si può proseguire nell'individuazione di una molteplicità di atti o vivenze, compito che ha accompagnato Husserl lungo l'arco di tutta la sua ricerca. La nostra coscienza, inoltre, registra vivenze relativi agli impulsi, agli istinti, alle tensioni, quelli cioè propri della sfera psichica, inoltre, quelli della decisione, della volontà delle prese di posizioni consapevoli i quali a loro volta costituiscono qualitativamente un altro raggruppamento e rimandano alla funzione spirituale. Fra i vivenze individuabili attraverso l'analisi ce n'è uno, in particolare, che è merito di Husserl aver evidenziato che è, appunto, l'atto dell'empatia. Testo della Stein.

Esaminando, allora, questo peculiare atto nel quale consiste la conoscenza dell'altro come conoscenza empatica Edith Stein si chiede che cosa si colga dell'altro e attraverso quale strumento ciò sia colto; il risultato dell'analisi rispetto a queste due questioni costituisce un'ulteriore conferma della complessità dell'essere umano, alla quale abbiamo accennato sopra. Se Husserl aveva condotto la sua indagine movendo prevalentemente dalla complessità e stratificazione della struttura umana in quanto tale, la sua discepola ricava le connotazioni fondamentali dell'antropologia, in primo luogo, proprio attraverso l'analisi dell'empatia. 


\section{Aoristo)))))}

\section{International Journal of Phenomenology, Hermeneutics and Metaphysics}

Ella scopre l'essere umano come individuo psicofisico; infatti, da un lato è coinvolta la corporeità come tramite necessario della conoscenza stessa, d'altro lato, la corporeità è animata, vivente $(L e i b)$, sede di affetti, di pulsioni, ma anche di pensieri, di ragionamenti, di decisioni e di volizioni. Questi atti sono qualitativamente uguali, possono essere posti senza distinzione sullo stesso piano o rimandano a gradualità diverse, implicanti anche valori diversi? In altri termini, qual è la genesi dell'interpretazione tradizionale secondo la quale sono rintracciabili "anima" e "corpo"? E, quindi, in quale senso l'essere umano non è riducibile alla corporeità, pur essendo questa la dimensione indispensabile della vita così come si dà, cioè come la costatiamo?

$E^{\prime}$ sul terreno delle vivenze di cui abbiamo coscienza, che s'individuano, come si è già notato, fondamentalmente le affinità, che si costituiscono i raggruppamenti i quali rimandano alle configurazioni, consolidate da una lunga tradizione e presenti anche se in forme diverse in tutte le culture, indicate, appunto, con i termini "corpo" e "anima". Il dolore o la gioia che l'altro vive deve essere colto, in primo luogo, attraverso una percezione legata agli organi della sensibilità, ma ciò che si scorge sul suo volto rimanda a una profondità di vita tale che è impossibile assimilarlo a qualsiasi oggetto fisico, visto e toccato. La dimensione che viene così scoperta è il luogo degli affetti, delle pulsioni, delle emozioni, è quella che indichiamo con il termine psiche (Psiche).

Ci si potrebbe chiedere - e Edith Stein ripropone una questione già indicata dal maestro - se non sia stata trovata ormai - siamo nel secondo decennio del Novecento - una disciplina che indaghi la psiche, che ce la mostri veramente come essa è, scientificamente, riducendola alla sua struttura che è di tipo meccanicistico: azione e reazione, associazione e così via, come sembrava sostenere la nascente psicologia, facendo proprio il metodo delle scienze della natura. Da questa domanda nasce il lungo e articolato saggio - pubblicato nel 1922 sull'“ Annuario" di fenomenologia diretto da Husserl - relativo a Psicologia e scienze dello spirito. Contributi per una fondazione filosofica (STEIN, 1999).

L'intento non è quello di eliminare tale disciplina in favore di una ricerca puramente filosofica, ma, piuttosto, di mostrare l'insufficienza del punto di vista della psicologia e delle nuove scienze umane, costituitesi nella seconda metà dell'Ottocento, in particolare la sociologia. Tale insufficienza riguarda sia la comprensione approfondita dell'essere umano sia la giustificazione della loro costituzione in quanto scienze; esse, infatti, hanno bisogno di un'indagine preliminare che chiarisca i momenti di fondo sui quali poggiano. Ad esempio, la psicologia non può fare a meno di chiedersi che cosa sia la psiche e deve anche domandarsi se corporeità e psiche siano sufficienti per comprendere l'essere umano nella sua interezza, e tali questioni rimandano a una riflessione filosofica.

Attraverso l'analisi dei vivenze Edith Stein mette in risalto l'esistenza di una sfera psichica caratterizzata da una "forza vitale", da un'energia psichica che svolge un ruolo fondamentale per la stessa sopravvivenza e capacità di agire dell'essere umano. Essa è sì strutturata secondo una sorta di causalità, in quanto sede di legami che, in una certa misura, possono essere ritenuti di tipo deterministico, ma è 


\section{Aoristo)))))}

International Journal of Phenomenology, Hermeneutics and Metaphysics

veramente tale da mostrarsi come l'unica fonte di direzione dell'essere umano stesso? Freschezza o stanchezza della psiche, ad esempio, sono sempre ed esclusivamente alla base delle nostre azioni e, quindi, delle nostre decisioni oppure le scelte ci rimandano a "motivazioni" che rivelano un fonte diversa di decisione? Ecco affacciarsi la sfera dello spirito (Geist).

L'empatia era già stata individuata come lo strumento di comprensione di ciò che è presente nella vita dell'altro e non solo relativamente ai suoi sentimenti o alle sue emozioni, ma anche relativamente al suo mondo interiore di decisioni, di volizioni, al suo mondo della creatività. Se ci sono le scienze dello "spirito", esse riguardano, appunto, ciò che è connesso con questa sfera che è quella della produzione culturale, artistica, politica e così via. Da un punto di vista filosofico, la presenza nell'essere umano della dimensione dello spirito consente di affermare che è superata la realtà psico-fisica che ci avvicina al mondo animale grazie ad un elemento che fa definire l'essere umano "persona".

Quest'interpretazione rimarrà fondamentale per l'Autrice anche quando la sua ricerca si rivolgerà al pensiero antico e medievale, anzi troverà in esso una conferma delle sue analisi fenomenologiche. I risultati ottenuti confluiscono nelle opere $\mathrm{La}$ struttura della persona umana (1932) (STEIN, 2013) ed Essere finito e Essere eterno (1936) (STEIN, 1994). In quest'ultima Edith Stein si sofferma, infatti, a indagare gli esseri finiti; ora fra questi si trova l'essere umano ed esaminandolo costata che "il suo corpo" è un corpo materiale; però, non solo questo, giacche è nello stesso tempo, anche un organismo che prende forma e agisce dall'intimo, e ancora: l'uomo non è solo organismo, ma è un essere vivente animato, che in modo particolare - percettivamente è aperto a sé e al mondo che lo circonda, e finalmente un essere spirituale, che è aperto conoscitivamente a se stesso e al resto, e può liberamente plasmare sé e il resto $\mathrm{e}$, in quanto tale, possiamo aggiungere sulla base di altre indicazioni fornite da Edith Stein, è "persona". Si può notare che il termine "persona" non solo è presente nelle analisi di Husserl e della Stein, ma si riferisce per ambedue alle stesse caratteristiche essenziali dell'essere umano, soprattutto alla sua componente spirituale.

\section{HUSSERL E I "PROBLEMI ULTIMI E SOMMI”}

Si è già notato che in riferimento alla persona e alla sua attività spirituale, Husserl parla dei problemi etico-religiosi e metafisici come i problemi ultimi e sommi. Il termine "metafisica" ha molti significati in Husserl: ci fermiamo solo sulla distinzione da lui proposta fra una "metafisica storicamente degenerata" e una "metafisica che in origine era stata fondata come filosofia prima"(HUSSERL, 2017, p. 191) intendendo egli con la prima un'indagine che si basa su un "eccesso di speculazione" e con la seconda l'autentico spirito della filosofia greca, riprendendo $l^{\prime}$ espressione aristotelica. Tuttavia, il riferimento non è qui direttamente a Platone o ad Aristotele, ma alla metafisica di Leibniz, in particolare, alla nozione di "monade". Il mio ego come monade, " non può a priori essere un ego che ha esperienza del mondo se non in quanto egli si trova in comunità con altri a lui simili, in quanto è un 


\section{Aoristo)))))}

International Journal of Phenomenology, Hermeneutics and Metaphysics

membro di una società di monadi che è orientata a partire da lui. [...] La dimostrazione conseguente di sé che compie il mondo oggettivo dell'esperienza implica una pari dimostrazione delle altre monadi come esistenti" (HUSSERL, 2017, p. 191).

Il che vuol dire che esiste un solo mondo oggettivo che è individuabile attraverso quella che egli definisce metafisica trascendentale, che mostra come, movendo dal soggetto, si posso comprendere la costituzione di un mondo oggettivamente esistente: ho chiamato quest'atteggiamento di Husserl, che sembra mettere insieme termini considerati tradizionalmente contrapposti come ego trascendentale ed esistenza oggettiva del mondo "realismo trascendentale" (ALES BELLO, 2014).

Husserl ritiene, in tal modo, - ma ciò che si è detto dovrebbe essere ulteriormente esplicitato e questo non è il luogo per farlo - che di rispondere all'obiezione secondo la quale: "[...] la fenomenologia non sarebbe capace di tanto poiché, partendo dall'ego trascendentale e dalla riduzione trascendentale, vi rimarrebbe legata, di modo che cadrebbe, senz'averne coscienza, nel solipsismo trascendental [...]" (HUSSERL, 2017, p. 200). Poiché tali argomentazioni si trovano nella $\mathrm{V}$ Meditazione cartesiana, Husserl mostra che è proprio il passaggio all'alterità attraverso la sua consistenza corporale che garantisce un mondo oggettivo esistente e che questa costatazione può fondare un realismo "trascendentale". Il nuovo punto di vista metafisico proposto da Husserl è da lui considerato valido, perché non si tratta di una costruzione "metafisica" legata solo a una tradizione, ma di una posizione giustificata sulla base dell'intuizione, che tende a "[...] esporre il senso che questo mondo ha per noi prima di ogni considerazione filosofica, senso che esso riceve in base alla nostra esperienza e che la filosofia può solo rivelare ma non mai mutare" (HUSSERL, 2017, p. 203).

Su questa linea analitica, che tende a porre l'accento sul senso della realtà esistente nella quale ci troviamo immersi, definita da Husserl "metafisica" potremmo aggiungere - delle realtà immanenti: io e mondo, egli procede a una metafisica della trascendenza assoluta.

Ho individuato sei " vie argomentative" che portano a Dio negli scritti di Husserl: la via "oggettiva" della finalità che ricorda la quinta via teleologica di Tommaso d'Aquino, la via soggettiva che muove da Agostino e Anselmo, la via intersoggettiva, quella leibniziana, appunto, che conduce alla Monade Somma, la via attraverso la hyletica, che è sempre una via teleologica che parte da dimensioni profonde della realtà, preriflessive e precategoriali, mostrando la presenza di un ordine delle cose, la via attraverso l'etica e forse una via "mistica" che approfondisce la presenza di Dio nell' interiorità (ALES BELLO, 2005)9.

Non potendo ripercorrere tutte queste vie nel presente scritto, mi fermo brevemente sulla via che conduce alla Monade Somma per continuare con coerenza

\footnotetext{
${ }^{9}$ Trad. inglese di A. Calcagno, The Divine in Husserl and Other Explorations, Analecta Husserliana, Springer, Dordrecht 2009; tr. spagnola di L. Rabanaque y M. Calello, Edmund Husserl. Pensar deus, creer Deus, Editorial Biblios- Paideia Fenomenológica, Buenos Aires 2016; tr. portoghese di A. Turolo Garcia, M. L. Fernandes, T. Akira, Edmund Husserl. Pensar Deus, Creer Deus, Paulus, São Paulo, 2016.
} 


\section{Aoristo)))))}

International Journal of Phenomenology, Hermeneutics and Metaphysics

sulla scia della costituzione metafisica monadica dell'io e del mondo. Se noi siamo il punto di partenza della ricerca e, quindi, costituiamo una sorta di assoluto quoad nos, Husserl scrive in un testo del 1922: "Da questo assoluto ci deve essere, allora, una via che conduce all'ultimo assoluto in un altro senso, da questo sistema di "sostanze" " in senso vero" (come essenti che non presuppongono l'essere che le costituisce) ci deve essere una via all'assoluta sostanza in senso ultimo" (HUSSERL, 1973).

$\mathrm{Si}$ è detto sopra che la posizione di Husserl non è sostanzialistica, in questo testo appare il termine sostanza che probabilmente è una semplice ripresa dell'uso leibniziano di definire le monadi sostanze. Husserl continua facendo esplicito riferimento alla Monade Somma come punto d'arrivo della via dell'intersoggettività a Dio, ciò è presente in particolare in un altro testo del 1922 intitolato Possibilità della fusione delle monadi. Possibilità di una Monade Somma (divina) (HUSSERL, 1922, p. 300302).

Non ci si può fermare sui temi etico-religiosi presenti nell'opera di Husserl, osservo solo che la trattazione di Husserl dei temi etici è in qualche caso connessa con i temi religiosi ed è presente, come ho detto sopra, anche una via etica che conduce a Dio. Husserl non crede che ci sia un cieco destino, al contrario, Dio regge il mondo e lo indirizza verso valori assoluti che appellano la libertà e la volontà umana sostenuta anche dalla grazia, per questo crede che ci sia Dio: "[...] la fede è un'esigenza assoluta e la più alta" (HUSSERL, 1924, p. 16).

Non a caso si tratta di un manoscritto non pensato per la pubblicazione; qui il problema metafisico che riguarda l'Assoluto s'intreccia con quello etico-religioso, in questo caso Husserl più che procedere a una rigorosa argomentazione espone i suoi pensieri più profondi e personali.

\section{EDITH STEIN E LE QUESTIONI METAFISICHE}

La Stein passa in modo non traumatico dall'analisi fenomenologica a una determinazione metafisica. Il soggetto non è solo io puro, io psichico e io spirituale, ma anche "sostanza". Il suo essere-separato è afferrabile dalla sua coscienza e diverso da qualsiasi altra cosa, può chiamare io solo se stesso ${ }^{10}$. Tuttavia, ella rimane sul terreno fenomenologico, quando riprende $\mathrm{i}$ temi dell'intenzionalità, temporalità, della vita spirituale come vita intellettiva, sulla connessione fra interiorità ed esterioritàn ${ }^{11}$.

Lo stesso accade per la vita morale, in cui emerge l'altra potenza dello spirito, cioè la volontà. Gli esseri umani si aprono, in tal modo, al mondo esterno, con tutta la loro persona che comprende la corporeità, la psiche (piacere e dispiacere), i sentimenti (amore e odio), la tonalità emotiva; tutto converge dal lato soggettivo alla formazione del carattere, che è legata anche alle caratteristiche proprie dell'individualità. La mappa complessa, stratificata, della vita interiore è completata

\footnotetext{
${ }^{10}$ Ivi, Cap. V, § 2.

${ }^{11}$ Ivi, Cap. V, §§ 4.5, 6.
} 


\section{Aoristo)))))}

International Journal of Phenomenology, Hermeneutics and Metaphysics

dalla presenza del nucleo, già individuato negli scritti fenomenologici della Stein e da lei definito ora anima dell'anima, punto profondo di convergenza dell'essere umano e luogo di apertura verso la trascendenza, costituita dagli altri e da Dio.

Con il sostegno di Tommaso lo spirito (Geist) si rivela sempre di più la sede delle operazioni intellettuali e volontarie nel doppio versante conoscitivo e morale. Tutto ciò era già stato detto da Husserl e dalla stessa Stein, ma qui si fonda su una base metafisica. E il nucleo (Kern) della persona, dal punto di vista di quest'ontologia rivisitata, acquista un'ulteriore determinazione sulla scia delle analisi già compiute nelle prime opere fenomenologiche (si veda soprattutto Psicologia e scienze dello spirito e La struttura della persona umana).

Per quanto riguarda la questione della conoscenza e della dimostrazione dell'esistenza di Dio, l'atteggiamento della Stein è peculiare. Ella ripete più volte che le "prove", le "dimostrazioni" non fanno trovare la fede; esse convalidano la fede, ma non conducono alla fede. Il credente, cioè colui che ha già una fede, intesa come conoscenza di Dio, si lascia condurre dalle prove, il non-credente rimane ai confini (STEIN, 1994, p. 150).

Questo non vuol dire che la fede sia un fatto irrazionale, al contrario, ella ritiene che la fides che giunge fino all'accettazione della Rivelazione possa, anzi debba, essere d'aiuto alla ragione naturale nella conoscenza della verità, in questo senso la ragione, illuminata dalla fede, può diventare una "ragione soprannaturale" espressione che ella usa solo in occasione del confronto fra Husserl e san Tommaso (STEIN, 2007), ma che, nel suo senso ultimo, mantiene sempre presente, perché questo serve a delimitare i confini della ragione umana, ma anche ad accettare la possibilità del suo potenziamento proprio attraverso la fede. D'altra parte, se la filosofia vuole raggiungere la verità, deve riconoscere le diverse fonti della verità e fra queste c'è la Rivelazione, che ha bisogno della fides come conoscenza naturale per essere accolta.

Tale circolarità è tenuta da lei presente quando in Essere finito e Essere eterno ella riflette sul significato di "Essere eterno" (STEIN, 1994).

Movendo da due testi della scrittura, Edith Stein osserva che essi ci portano più lontano di quanto possa raggiungere una ricerca intellettuale. Si tratta dell'inizio del Vangelo di Giovanni "In principio era il Logos" e del passo della lettera di San Paolo ai Colossesi 1, 17, in cui si dice che: "Egli è prima di tutte le cose e tutte la cose hanno in lui la loro consistenza e connessione". E aggiunge che forse il significato filosofico del Logos, al quale siamo rimandati, può aiutarci a capire il significato teologico e, a sua volta, la verità rivelata può risolvere le difficoltà filosofiche. Procede, pertanto, ad una chiarificazione filosofica dei due testi.

A suo avviso i due testi rimandano alla visione Trinitaria di Dio. In particolare: ev arche ev o logos ha questo significato: nell'Ente primo era incluso il Logos (il senso o l'essenza divina), nel Padre era il Figlio, cioè il "senso" dell'attualità originaria; quindi, la generazione significa la posizione dell'essenza nella nuova attualità personale del Figlio, che, tuttavia, non è una posizione all'esterno dell'attualità originaria del padre. Nel distinguere, però, l'essere essenziale, dall'essere attualereale si corre un rischio di separarlo nel pensiero e ciò non è legittimo. L'autrice qui 


\section{Aoristo)))))}

\section{International Journal of Phenomenology, Hermeneutics and Metaphysics}

sottolinea fortemente la validità della posizione di san Tommaso, il quale insiste sull'inseparabilità fra l'essenza e l'essere attuale-reale nel primo Ente, cosa che distingue l'Essere da qualsiasi altro ente finito in cui è possibile operare tale distinzione.

Ella, proseguendo nell'indagine, osserva che, poiché il primo Ente ha l'essere per essenza, è impossibile anche solo pensarlo senza l'essere, ma bisogna chiedersi quale sia la consistenza di tale "pensare". Se si riuscisse, infatti, a pensare veramente in modo riempiente - e qui la Stein adopera il linguaggio fenomenologico, intendendo il riempimento totale di un'intenzione vuota - saremmo di fronte alla comprensione profonda di quella che Anselmo proponeva come "prova" dell'esistenza di Dio.

Ella riflette, però, ulteriormente su tale questione, sostenendo che, se riuscissimo a cogliere "veramente" la coincidenza fra essere ed essenza, non saremmo di fronte ad una "prova" e, quindi, dal punto di vista logico, ad una "conclusione" o deduzione, ma avremmo dato una forma al "pensiero originario" ("eine Umformung des ursprüngliches Gedankens"), relativo a quella coincidenza, lo avremmo "foggiato". E aggiunge: "L'esattezza di questa messa in forma non è contestata neppure da Tommaso d'Aquino, il quale, com'è noto, ha respinto la prova ontologica" (STEIN, 1994, p.149).

Anche Tommaso, in fondo, afferma che la proposizione "Dio esiste" è evidente in sé, ma non per noi e, proprio perché non riusciamo a cogliere veramente questa evidenza, abbiamo bisogno di una dimostrazione dagli effetti. Pure in questo modo, tuttavia, non arriviamo alla comprensione e qui, in ultima analisi, secondo Edith Stein, ha ragione Agostino quando afferma: "Si comprehenderis non est Deus". Anselmo, che si sforza di dare una forma al pensiero originario, fallisce, perché non arriva alla comprensione piena.

La ragione profonda del fallimento da parte di tutte le prove o le vie di raggiungere il riempimento senza riserve sta nella tensione irrisolvibile dello spirito umano tra finito e infinito. E questo ci fa capire anche il destino singolare della prova ontologica, secondo Edith Stein: che si trovino sempre nuovi difensori e avversari di essa.

Non è possibile, quindi, cogliere attraverso la via della conoscenza naturale la coincidenza fra essenza ed esistenza. Per chi ha fede sembra impossibile che non ci sia questa coincidenza, ma quando cerca di comprenderla intellettualmente è destinato a fallire. Si è costretti solo ad avvicinarsi a Dio attraverso immagini finite, a volte dal lato dell'essenza e a volte dal lato dell'essere.

Com'è noto, la Stein sostiene che una conoscenza piena di Dio, conformemente alle sue capacità che sono sempre limitate in quanto è creatura, è possibile all'essere umano solo nell'esperienza mistica, perché Dio eleva a sé l'anima e le fa cogliere in modo intuitivo il senso di tutte cose, secondo la testimonianza di Santa Teresa d'Avila (STEIN, 1997). Perciò l'esperienza mistica illumina la conoscenza di sé ed è di grande aiuto per la comprensione della struttura antropologica e di Dio. 


\section{Aoristo)))))}

International Journal of Phenomenology, Hermeneutics and Metaphysics

I temi metafisici, pur affrontabili in modo autonomo rispetto alla fede e alla visione mistica, possono essere illuminati da questi tipi peculiari di "conoscenze" e più facilmente affrontati.

\section{CONCLUSIONE}

Come si è cercato di mettere in evidenza, i filosofi ai quali ho fatto riferimento non si sottraggono alle grandi domande riguardanti: chi sono io? Qual è il senso della realtà a noi più vicina e della realtà ultima? Con minore o maggiore vicinanza rispetto alle posizioni classiche e costruttive della storia della filosofia e in colloquio con l'Età Moderna (Husserl) o con il pensiero antico e medievale (Stein), attraverso un'impostazione originale e convincente, mostrano come tali domande non siano eludibili e come a esse sia possibile dare una risposta valida.

\section{REFERENZE}

ALES BELLO, A. Edmund Husserl: Pensare Dio Credere in Dio. Pandova: Messaggero, 2005. . Human World - Animal World: An Interpretation of Instinct in some late Husserlian Manuscripts. In: Analecta Husserliana. V. LXVIII (2000). . Il senso delle cose. Per un realismo trascendentale. Roma: Castelvecchi, 2014.

HEIDEGGER, M. Essere e tempo. Trad. P. Chiodi. Milano: Bocca, 1953.

HUSSERL, E. Ethisches Leben, Theologie. Wissenschaft. Ms AV 21, 1924.

. Die transzendentale Alter Ego gegenüber der Transzendenz der fremden Subjektivität des

Dinges. Absolute Monadologie als Erweiterung der transzendentalen Egologie. Absolute

Weltinterpretation, (Januar/Februar 1922). In: Zur Phänomenologie der Intersubjektivität II,

Husserliana Bd. XIV, hrsg. von I .Kern. Dordrecht: Martinus Nijhoff, 1973.

Idee per una fenomenologia pura e una filosofia fenomenologica. Vol. I. Trad. V. Costa. Torino:

Einaudi, 2002.

. La crisi delle scienze europee e la fenomenologia trascendentale. Trad. E. Filippini. Milano: II

Saggiatore, 1987.

. Meditazioni cartesiane e Lezioni parigine. (Org.) A. Canzonieri. Trad. V. Costa. Morcelliana: La

Scuola, 2017.

. Möglichkeit der Verschmelzung der Monaden. Möglichkeit einer (göttlichen) Übermonade

(1922). Beilage XLI. Husserliana Bd. XIV, hrsg. von I .Kern. Dordrecht: Martinus Nijhoff, 1973.

. Phänomenologie und Anthropologie. In: Aufträge und Vorsätze (1922-1937), Husserliana, Bd. XXVII.

. Phänomenologie und Psychologie. In: Aufsätze und Vorträge I, Husserliana Bd. XXV, hrsg. von Th. Nenon and H. R. Sepp. Dordrecht: Martinus Nijhoff, 1987.

STEIN, E. Essere finito e Essere eterno. Per un'elevazione al senso dell'essere. Trad. L. Vigone, Rev. E Presentazione di A. Ales Bello. Roma: Città Nova, 1994.

. Il Castello interiore. In: Natura Persona Mistica. Per una ricerca cristiana della verità. (Org.) A.

Ales Bello; Trad. A. M. Pezzella. Roma: Città Nuova, 1997.

. IL problema dell'empatia. Trad. E. Costantini e E. Schulze; (Prefazione di A., Ales Bello). Roma:

Studium, 1998.

. La fenomenologia di Husserl e la filosofia di san Tommaso d'Aquino. Trad. Angela Ales Bello, In:

Edith Stein, La ricerca della verità - Dalla fenomenologia alla filosofia cristiana, a cura di Angela Ales

Bello, Roma: Città Nuova, 2007 (ristampa). 


\section{Aoristo)))))}

International Journal of Phenomenology, Hermeneutics and Metaphysics

. La struttura della persona umana. Corso di antropologia filosofica. Trad. M.D'Ambra, revisione di A.M. Pezzella, M. Paolinelli; (Prefazione di A. Ales, a cura di A. Ales Bello e Marco Paolinelli). Roma: Città Nuova/Edizioni OCD,2013. La struttura della persona umana. Trad. M. D’Ambra; Rev. di A. M. Pezzella e M. Paolinelli, a cura di A. Ales Bello e M. Paolinelli, Roma: Città Nuova, 2013. Psicologia e scienze dello spirito. Trad. M. Pezzella; Roma: Città Nuova, 1999.

Inserito: 4 giugno 2021

Accettato: 1 luglio 2021 